\title{
SLDR: a computational technique to identify novel genetic regulatory relationships
}

\author{
Zongliang Yue ${ }^{2,4}$, Ping Wan ${ }^{4}$, Hui Huang ${ }^{2}$, Zhan Xie ${ }^{4}$, Jake Y Chen ${ }^{1,2,3^{*}}$ \\ From 11th Annual MCBIOS Conference \\ Stillwater, OK, USA. 6-8 March 2014
}

\begin{abstract}
We developed a new computational technique called Step-Level Differential Response (SLDR) to identify genetic regulatory relationships. Our technique takes advantages of functional genomics data for the same species under different perturbation conditions, therefore complementary to current popular computational techniques. It can particularly identify "rare" activation/inhibition relationship events that can be difficult to find in experimental results. In SLDR, we model each candidate target gene as being controlled by $N$ binary-state regulators that lead to $\leq 2^{\mathrm{N}}$ observable states ("step-levels") for the target. We applied SLDR to the study of the GEO microarray data set GSE25644, which consists of 158 different mutant $S$. cerevisiae gene expressional profiles. For each target gene $t$, we first clustered ordered samples into various clusters, each approximating an observable step-level of $t$ to screen out the "de-centric" target. Then, we ordered each gene $x$ as a candidate regulator and aligned $t$ to $x$ for the purpose of examining the step-level correlations between low expression set of $x\left(\mathrm{R}_{\mathrm{o}}\right)$ and high expression set of $x\left(\mathrm{R}_{\mathrm{h}}\right)$ from the regulator $x$ to $t$, by finding $\max f(t, x):\left|\mathrm{R}_{\mathrm{o}}-\mathrm{R}_{\mathrm{h}}\right|$ over all candidate $\times$ in the genome for each $t$. We therefore obtained activation and inhibitions events from different combinations of $R_{o}$ and $R_{h}$. Furthermore, we developed criteria for filtering out less-confident regulators, estimated the number of regulators for each target $t$, and evaluated identified topranking regulator-target relationship. Our results can be cross-validated with the Yeast Fitness database. SLDR is also computationally efficient with $\mathrm{o}\left(\mathrm{N}^{2}\right)$ complexity. In summary, we believe SLDR can be applied to the mining
\end{abstract}

\footnotetext{
* Correspondence: jakechen@iupui.edu

${ }^{1}$ Institute of Biopharmaceutical Informatics and Technology, Wenzhou Medical University, Wenzhou, Zhejiang Province, China

Full list of author information is available at the end of the article
}

of functional genomics big data for future network biology and network medicine applications.

\section{Introduction}

Identifying novel gene regulatory relationship from large-scale functional genomics data has been a major theme for the characterization of complex biomolecular systems. Gene regulator identification can be identified from gene expression data using DNA microarrays. With tens of thousands of microarray experiments deposited into public databases for yeast, Drosophila, Arabidopsis, mice, and humans, one may reconstruct molecular interaction or regulation relationships from mining the data without conducting specific experiments to test whether a candidate regulator-target relationship exists. For example, James et al [1] examined temporal gene expression patterns during chondrogenic differentiation in a mouse micromass culture system. Then, they determined transcriptional regulation by observing the impact of changed expression of molecules onto changed gene functional categories. Lorenz et al [2] used microarray analysis and scale-free gene networks analysis to identify candidate regulators in drought-stressed roots of loblolly pine. Systematic approaches to reconstruct transcriptional modules and identify their perturbation conditions are under way [3]. Albert et al [4] summarized recent findings that the disruption of regulatory relationships may lead to human diseases, therefore shedding new light on disease intervention on gene regulatory relationships instead of genes as possible drug targets-hence the new field of "network medicine". These examples show a surging interest among genome biologists to study gene regulatory relationships.

Traditional experimental gene regulator finding methods, e.g., those using gene knockouts, synthetic lethality, or chip-seq in eukaryotes, are too costly to serve as the 
primary platform with which scientists explore the large combinatorial space between all candidate pairs of genes [5-7]. To overcome the data coverage gap, many computational methods have been proposed, e.g., homologous gene regulator database search, clustering of gene expression profiles and transcription factor binding site pattern matching, physics-based modeling of candidate transcription factors and target binding relationships, and network based methods [4,8]. For example, Ru-Fang Yeh et al [9] introduced an accurate and efficient technique that performs homologous gene regulator database search in higher eukaryotes to annotate gene regulators for the human genome. Stephane et al [10] developed a rigorous statistical test to establish a link between selection threshold of putatively regulators and the identified false positive genes in clusters of candidate gene targets derived from gene expression profiles. Gerhard et al [11] developed ANREP, a system that can identify exact pattern matches to motifs with spacing constraints and approximate matches recursively. Physics-based methods that characterize protein-ligand relationships, e.g., the MM-based methods, have also been proposed [12].

In this study, our aim is to develop a new computational method to identify genetic regulatory relationships that are difficult to uncover using previously reported techniques. This type of relationships differ from gene regulatory relationships in that genes in the former type may affect each other indirectly through other genes or molecular regulation mechanisms while genes in the latter type affect each other as direct, observable regulator-target relationships. Current databases often cover reasonably well highlyconnected gene regulators, or "hubs" of gene regulations in the gene regulatory network [13]; however, for lowconnectivity regulators, or "de-centric nodes" in the gene regulatory network, the coverage is often poor because the chance for randomly observing their activities is low. Step-Level Differential Response (SLDR) is a new computational method developed to identify de-centric genetic regulatory relationship candidates. The input of SLDR is the functional genomics data under permutated perturbation conditions. In SLDR, we specifically search for all qualifying target genes, each which is controlled by $N$ binary-state regulators that lead to $\leq 2^{\mathrm{N}}$ observable expression levels-which we call "step-levels"-of the target gene. The output of SLDR is statistically significant findings candidate genetic regulatory relationships. We describe our study in detail next.

\section{Method}

\section{An overview of the framework}

We show an overview of workflow of the SLDR data analysis framework (Figure 1). First, the expression values in perturbed gene expression data sets will be averaged across biological independent replicates and groups without mutation will be filtered off. Second, decentric genetic regulatory relationship targets will be selected after clustering of gene expression profiles. Third, a statistical correlation-based model will be applied to the extraction of significant de-centric activation/inhibition relationship pairs and a threshold will be applied to the rejection of low-confidence de-centric genetic regulatory relationship pairs. Fourth, the de-centric genetic regulatory network thus generated will be assessed with network "index of aggregation" test. Fifth, novel candidate genetic regulatory pairs will be evaluated with several public gene regulation databases and a hyper-geometric test will be used to rank the top 10 suspected de-centric targets predicted by SLDR. Sixth, the robust of the de-centric networks will be tested by performing the shuffle method to introduce noise. Seventh, samples will be clustered, which is significantly contribute to the de-centric targets. Additionally, the de-centric networks' function will be analyzed by Gene Ontology and sub-cellular localization. The comprehensive output of SLDR analysis can consist of: a distribution curve of target by genetic regulator number, two networks of activation/inhibition de-centric genetic regulation, and a list of the top 10 suspected de-centric genetic regulatory pair candidates.

\section{Preparation of functional genomics data}

We used raw data GSE25644 from the Gene Expression Omnibus (GEO) database (http://www.ncbi.nlm.nih.gov/ geo/) as the input. GSE25644 is a DNA microarray gene expression profile with all 158 viable protein kinase/ phosphatase deletions in S. cerevisiae under a single growth condition [14]. Each mutant was profiled four times, from two independent cultures on dual-channel microarrays using a batch of wild-type (WT) RNA as a common reference. The GSE25644 was normalized by averaging each of the two independent cultures' results on microarrays, and before our algorithm was applied, the wild-type groups were filtered off.

\section{Selection of the de-centric targets}

The selection of de-centric targets is based on clustering of gene expression profiles. To find the potential decentric targets which be regulated with $\mathrm{N}$ regulators, we modeled $k^{\prime}+1 \leq 2^{N}$ for each candidate target gene as being controlled by $\mathrm{N}$ binary-state regulators that lead to k' observable states ("step-levels"). Here, we introduced $k$ ' which is the number of steps generated from each gene RNA expression. If there are sufficiently large collections of functional genomics experiments, each being performed under a heterogeneous perturbation condition, the method will search each target's genetic 


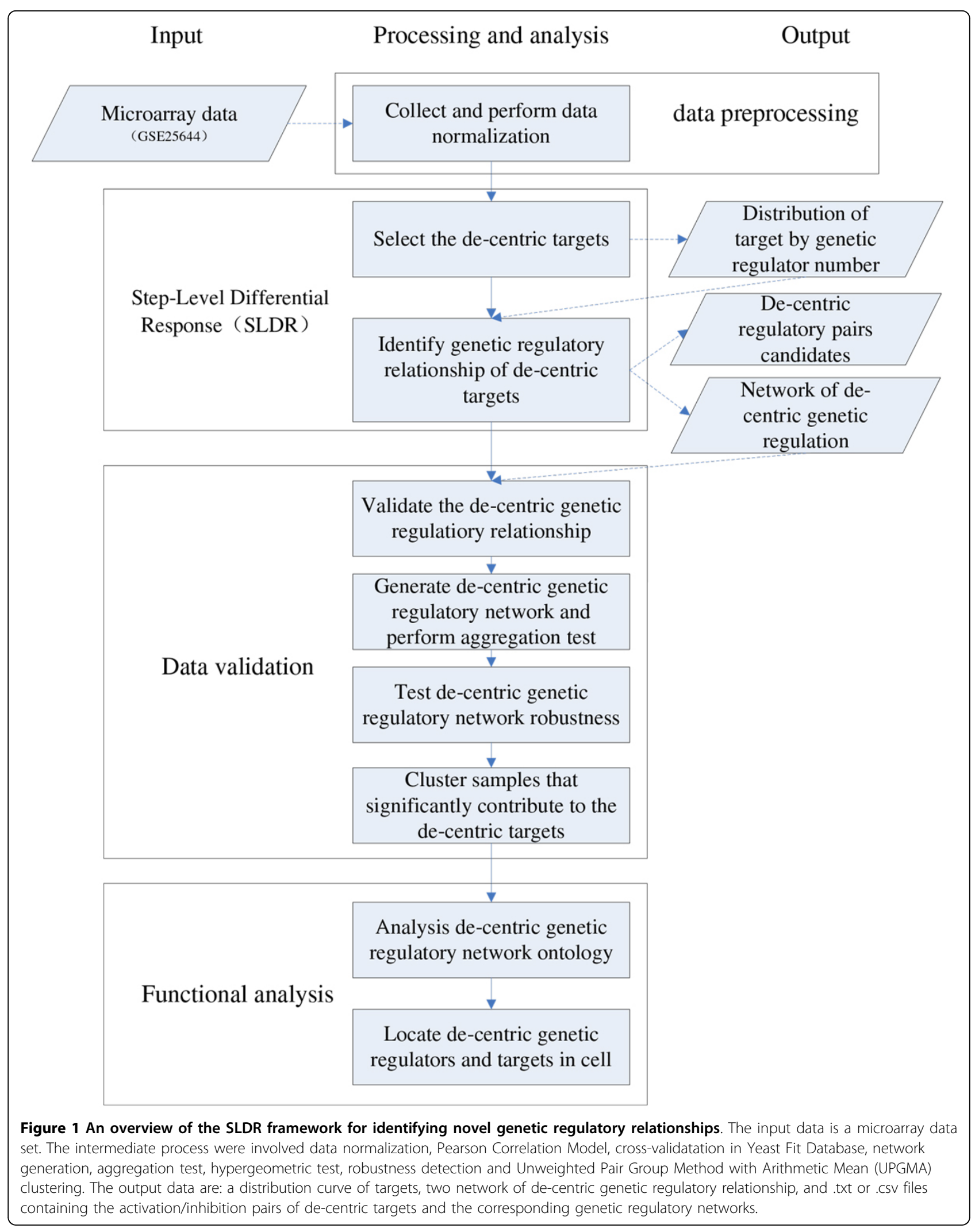


regulator candidates to test if a significant switchresponder pattern exists before ranking candidate genetic regulators (Figure 2).

1) In order to find out the huge steps, we use average steps as a standard to filter. The average increasing value $\Delta$ of each gene was calculated and averaged values were sorted. The average increasing value $\Delta$ was calculated by the maximum value of the gene expression (we assume the target gene $t$ ) minus the minimum value and then divided by the number of samples n. $\Delta_{\mathrm{t}}=\left(\mathrm{t}_{\max }-\mathrm{t}_{\min }\right) / \mathrm{n}$.

2) The average increasing value $\Delta_{t}$ of a target was regarded as a standard to seek steps which means the number of dj larger than $\Delta_{t}$, which we defined as $\mathrm{k}$ value. For each target, gene expression was sorted from low to high and then we calculated the difference between adjacent samples, $d j$. If the difference $d j$ is smaller than corresponding $\Delta \mathrm{t}$, $\mathrm{k}$ will be retained. The formula of step $k$ is shown below:

$\mathrm{dt}_{\mathrm{i}+1}-\mathrm{dt}_{\mathrm{i}}\left\{\begin{array}{c}\geq \Delta, k=k+1 \\ <\Delta, k=k\end{array}\right.$

3) Iteration was performed for every target to find out each $\mathrm{k}$ of targets. $\mathrm{k}$ values were sorted from low to high and the largest $k_{\max }$ corresponds to target $t^{\prime}$. 4) To avoid the situation that fake steps with small change causes high $\mathrm{k}$ ' in individual, every target was normalized by the new average increasing value $\Delta_{\max }$ as a standard to seek for new step levels (k') of each target. The formulas of new average increasing value $\Delta_{\max }$ and step k' are shown below:

$\Delta_{\max }=\left(\mathrm{t}_{\max }^{\prime}-\mathrm{t}_{\text {min }}^{\prime}\right) / \mathrm{k}_{\max } \mathrm{t}_{\mathrm{i}+1}-\mathrm{t}_{\mathrm{i}}\left\{\begin{array}{c}\geq \Delta_{\max }, k^{\prime}=k^{\prime}+1 \\ <\Delta_{\max }, k^{\prime}=k^{\prime}\end{array}\right.$
5) The binary state $\mathrm{N}$ was calculated, and $\mathrm{N}$ means the number of genetic regulators of each target. For instance, assuming that a target's binary state $\mathrm{N}$ is 2 , this target would have less than 3 steps within 4 step-levels theoretically. The formula of $\mathrm{N}$ binarystate is shown below:

$$
2^{\mathrm{N}}=\mathrm{k}^{\prime}+1
$$

We can use the cluster $\mathrm{k}^{\prime}$ to calculate the theoretical number of genetic regulators of the de-centric targets.

\section{Identification of genetic regulatory relationship among genes}

The genetic regulatory relationships of regulators to targets were predicted based on the expression pattern associated with Pearson Correlation. First, the model of activation/inhibition is determined by the low gene expression pattern and the high gene expression pattern in (Figure 3). Here we sorted the expression values of every gene regarded as regulator, then align the other potential targets to it. The regulator's lowest $20 \%$ expression was regarded as low expression set, and highest $20 \%$ expression was regarded as high expression sets since all of the de-centric steps located in these expression sets. When the low expression set has low information and high expression set has high information, we supposed that a activation or inhibition existed. For instance, if there exists a positive genetic regulator activating its target, the correlation in low gene expression's step-level would be low to 0 and the correlation in high gene expression's step-level would be high to 1 .

To explore the potential activation/inhibition genetic regulatory relationship between regulator $x$ and target $t$, each gene $t$ was regarded as a genetic target candidate was aligned to each step level of the regulator $\mathrm{x}$. The

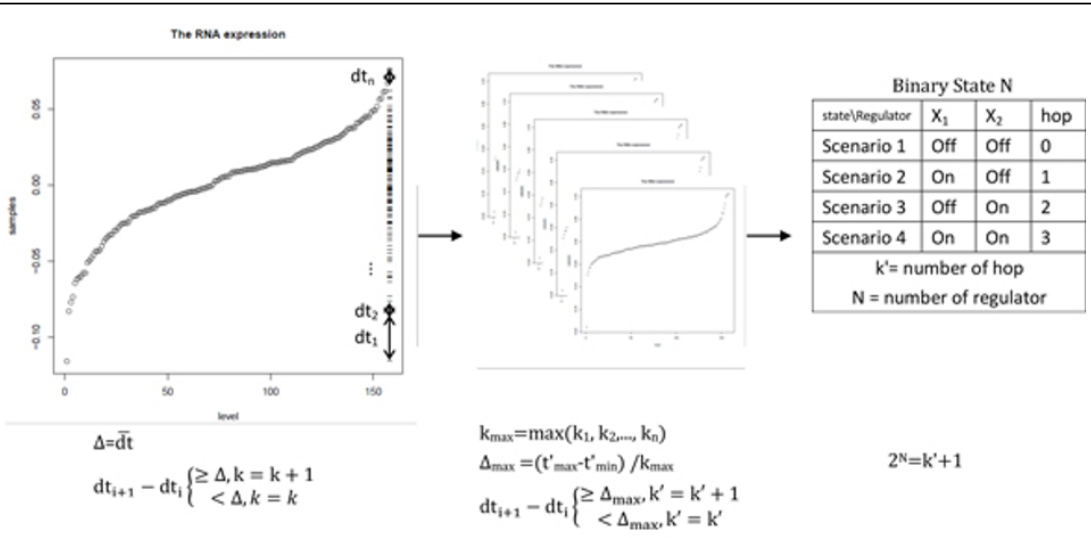

Figure 2 The workflow of de-centric targets generation. First, we used average changing value as standard to screen out the number of steps. Second, to normalize the steps, we use the largest steps gene as new standard to screen out the number of steps. Third, we apply binary states method to find out theoretical regulators' number according to the number of steps. 


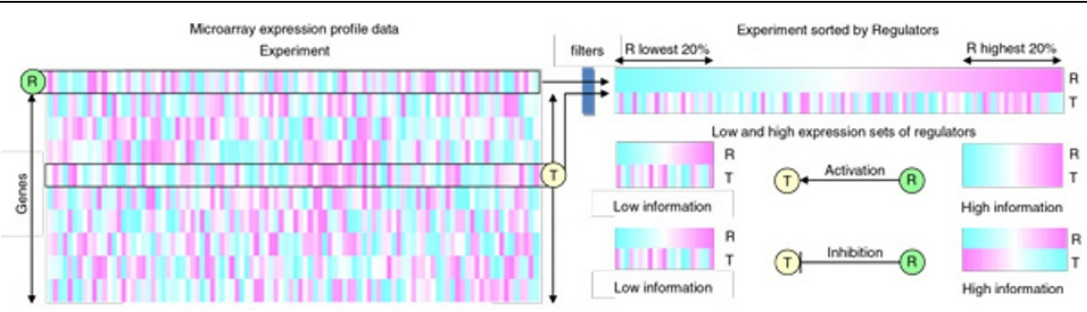

Figure 3 The model of activation/inhibition predicted based on the expression pattern associated with Pearson Correlation. A gene expression profile dataset was represented as a matrix, where columns indicated different samples and rows indicated different genes. The expression profiles of regulators were sorted, and the lowest $20 \%$ and highest $20 \%$ were regarded as low expression sets and high expression sets. The activation and inhibition of potential regulators had low information in low expression set and high information in high expression set.

low step levels of $t_{0}$ and the high step levels of $t_{h}$ were used to compute Pearson correlation $R_{0}$ and $R_{h}$ respectively. The Pearson correlation formula is shown below:

$$
\rho(\mathrm{x}, \mathrm{t})=\frac{\operatorname{cov}(\mathrm{x}, \mathrm{t})}{\rho \mathrm{x} \rho \mathrm{t}}=\frac{\mathrm{E}\left[\left(\mathrm{x}-\mu_{\mathrm{x}}\right)\left(\mathrm{t}-\mu_{\mathrm{t}}\right)\right]}{\rho \mathrm{x} \rho \mathrm{t}}
$$

Where, cov is the covariance between potential regulator $x$ and target $\mathrm{t}, \rho \mathrm{x}$ is the standard deviation of $\mathrm{x}, \boldsymbol{\mu}_{\mathrm{x}}$ is the mean of $x$, and $E$ is the expectation.

In order to get the strict result of inhibition and activation relationship, the high information and low information were designed as 0.8 and 0.2 . For instance, the $R_{o}$ located in $(-0.2,0.2)$ and the $R_{h}$ located in the $(0.8,1)$ indicated the $\times$ has positive genetic regulation on $t$. For illustrative purposes, we show a simple table (Table 1) to explicitly explain the activation/inhibition models of the genetic regulator $\times$ to target $t$.

To reject the incorrect genetic targets $t$, we required that the middle gene expression step-levels of the genetic targets $t$ shouldn't have a significant larger change than the average change values of gene expression. Assuming that one genetic target $t$ has one middle gene expression step-level from step $i$ to step $j$ and the lowest gene expression value among $t_{i}$ to $t_{j}$ is $t_{\text {low }}$, the change of gene expression from $t_{n}$ ( $n$ in $i$ to $j$ ) to $t_{\text {low }}$ shouldn't be more than the genetic regulator's average change level of the value $\Delta_{t}$. The formula of rejection criteria is shown below:

$$
\mathrm{t}_{\mathrm{n}}-\mathrm{t}_{\text {low }}<\Delta_{\mathrm{t}} *(\mathrm{j}-\mathrm{i}) \mathrm{n} \in(\mathrm{i}, \mathrm{j}) \text { while } \Delta_{\mathrm{t}}=\left(\mathrm{t}_{\max }-\mathrm{t}_{\min }\right) /\left(\mathrm{t}_{\text {size }}\right)
$$

To examine the step-level correlation between low step levels of the $x\left(\mathrm{R}_{0}\right)$ and high step levels of the $x$

Table 1 Representation of Activation/Inhibition genetic relationship pairs identified and the threshold used between $R_{0}$ and $R_{h}$ cases

\begin{tabular}{ccc}
\hline & $\mathbf{R}_{\mathbf{0}}$ & $\mathbf{R}_{\mathbf{h}}$ \\
\hline $\mathrm{x} \rightarrow(+) \mathrm{t}$ & $\rightarrow 0(-0.2,0.2)$ & $\rightarrow 1(0.8,1)$ \\
$\mathrm{x} \rightarrow(-) \mathrm{t}$ & $\rightarrow 0(-0.2,0.2)$ & $\rightarrow-1(-0.8,-1)$ \\
\hline
\end{tabular}

$\left(\mathrm{R}_{\mathrm{h}}\right)$ between the $x$ and $t$, all genetic regulator candidates were ordered to each target using $\max f(t, x):\left|\mathrm{R}_{\mathrm{o}}-\mathrm{R}_{\mathrm{h}}\right|$. Then we choose top 10 high $\left|R_{\mathrm{o}}-\mathrm{R}_{\mathrm{h}}\right|$ genetic regulatory pairs, since the number of nodes generating the genetic regulatory network should be balanced and moderate to present the de-centric targets. If the pairs are strict, they will not organize a network with enough pairs. If the pairs are relax, they will introduce noise of weak links in the networks.

\section{Generation of the de-centric genetic regulatory network and testing of network significance}

We generated the de-centric genetic regulatory network by the top 10 high $\left|R_{o}-R_{h}\right|$ genetic regulator pairs and performed statistical data analysis tests to detect the significance of the connected network. Our hypothesis of this statistical evaluation is that if the prediction model indeed consists of de-centric targets involved in the same process even if complex and broad, then we should expect that the connectivity among the de-centric targets be lower than the connectivity among a set of randomly selected genes.

We defined the index of aggregation of a network [8] as the ratio of the size of the largest sub-network that exists in this network to the size of this network. Note that the size is calculated as the total number of genes within a given network/sub-network.

To test the hypothesis that the predicted targets are less connected than a randomly selected set of targets, we developed the null hypothesis test using the following re-sampling procedure :

1) Randomly select from the pool of genetic regulators to targets, the same number of predicted targets generated from our method.

2) Retrieve the top 10 genetic regulators of each random target using $\left|R_{\mathrm{o}}-\mathrm{R}_{\mathrm{h}}\right|$ criteria.

3) Compute the index of the aggregation of superset.

4) Repeat steps 1 through 3 for 500 times to generate a distribution of the index of aggregation under random selection. 
5) Compare the index of aggregation from our method with the distribution obtained in step 4 and calculate the p-value.

\section{Significance testing of the de-centric genetic regulatory relationships}

Our result is validated in the Yeast Fitness Database (http://chemogenomics.med.utoronto.ca/fitdb/fitdb.cgi). FitDB is a searchable database of quantitative chemicalgenetic interactions based on data in Hillenmeyer [15]. A gene search allows viewing of the compounds that are most sensitive to the gene specified in a heterozygous and homozygous yeast deletion strain, including a view of yeast deletion strains that behave similarly to the gene of interest. Compounds can also be searched to identify heterozygous or homozygous deletion strains exhibiting hypersensitivity to compound, including a view of compounds that behave similarly to the compound of interest.

Hypergeometric test was introduced to see the significant of the top 10 high $\left|R_{0}-R_{h}\right|$ pairs in genetic regulator candidates. The validated number of the top 10 high $\left|R_{o}-R_{h}\right|$ pairs in Yeast Fit database was $k$. The total number of the top 10 high $\left|R_{o}-R_{h}\right|$ pairs is $n$. The validated number of randomly chosen pairs in Yeast Fit database is K. The total number of randomly chosen pairs is $\mathrm{N}$. The use of hypergeometric test is illustrated in (Table 2).

The variable number of top 10 high $\left|R_{o}-R_{h}\right|$ pairs $\times$ follows the hypergeometric distribution by its probability mass function (pmf) given by the formula below:

$$
P(X=k)=\frac{\left(\begin{array}{l}
K \\
k
\end{array}\right)\left(\begin{array}{l}
N-K \\
n-k
\end{array}\right)}{\left(\begin{array}{l}
N \\
n
\end{array}\right)}
$$

\section{Test de-centric genetic regulatory network robustness}

In order to detect the robustness, we introduced the noise on the gene expression profiles of the 158 viable protein kinase/phosphatase deletions strains. The noise was designed as increasing $5 \%, 10 \%, 15 \%, 20 \%, 30 \%$, $50 \%, 70 \%$ of noise by randomly shuffling the expression values of each sample.

Table 2 A contingency table showing how to perform the hypergeometric test

\begin{tabular}{rcc}
\hline & top 10 & random \\
\hline validated & $k$ & $K$ \\
un-validated & $n-k$ & $N-K$ \\
total & $n$ & $N$ \\
\hline
\end{tabular}

\section{Cluster the samples that significantly contribute to the} de-centric target

The 158 viable protein kinase/phosphatase deletions' profiles was clustered by UPGMA (Unweighted Pair Group Method with Arithmetic Mean). The agglomerative clustering method UPGMA is one of the most popular methods for the classification of sampling units on the basis of their pairwise similarities in relevant descriptor variables. We used UPGMA algorithm to construct a rooted tree that reflects the structure in a pairwise samples' similarity matrix.

At each step, the nearest two clusters are combined into a higher-level cluster. The distance between any two clusters $A$ and $B$ is taken to be the average of all distances between pairs of objects " $x$ " in A and " $y$ " in B, that is, the mean distance between elements of each cluster:

$$
\frac{1}{|A| \times|B|} \sum_{x \in A} \sum_{y \in B} d(x, y)
$$

Then we find a minimal threshold in the hierarchical tree and pick a representative cluster. Delete it to see the effect of the cluster on finding of de-centric targets.

\section{Analysis de-centric genetic regulatory network ontology}

In order to explore the function of the de-centric regulatory networks, ClueGo [16], a Cytoscape plug-in was used. ClueGO performs single cluster analysis and comparison of clusters. From the ontology sources used, the terms are selected by different filter criteria. The related terms which share similar associated genes can be fused to reduce redundancy. The ClueGO network is created with kappa statistics and reflects the relationships between the terms based on the similarity of their associated genes. ClueGO charts are underlying the specificity and the common aspects of the biological role. The significance of the terms and groups is automatically calculated.

\section{Locate the de-centric regulators and targets in cell}

The sub cellular location of de-centric regulators and targets were retrieved in the Comprehensive Yeast Genome Database [17], which derived from experiments, (CYGD: http://mips.gsf.de/genre/proj/yeast/) and display by the tool, Cerebral, a cytoscape plug-in. Cerebral uses sub cellular localization attribute to create a layered view of a cell, placing nodes in the region of the screen corresponding to the appropriate localization. [18]

\section{Results}

1. Determination of de-centric genetic regulated targets from the processed data

The distribution of the number of genetic regulators for each target follows Gaussian distribution which means 
the number of genetic regulators is a random variable independently drawn from the same distribution. 453 targets were selected, which has no more than two genetic regulators after the clustering of gene expression profiles. (Figure 4) According to the formula: $2^{\mathrm{N}}=\mathrm{k}^{\prime}+1$, the $\mathrm{N}$ was determined by the cluster $\mathrm{k}^{\prime}$ and all targets were clustered into 8 groups with $\mathrm{N}$ varying from 0 to 7. If the $\mathrm{N}$ was chosen as 1 , which means the theoretical target's genetic regulator equal to 1 , the thresholds was so strict that include 59 out of 6047 targets with coverage $1 \%$. If the $\mathrm{N}$ was chosen as 3 , the thresholds was so relax that include 2579 out of 6047 with coverage $43 \%$ nearly to half. Meanwhile, the mis-clustering will be severe with $k^{\prime}$ varied from 1 to 7 , which will be discussed afterwards. We chose the modest thresholds; $\mathrm{N}$ as 2 that include 453 out of 6047 with coverage $7.5 \%$ as shown in left shade region separated by red line which displayed the target's genetic regulator equal or below 2 excluding 0 .

The $2^{\mathrm{N}}=\mathrm{k}^{\prime}+1$ is the key point to control the theoretical genetic regulator number of targets. Especially the selection of binary-state $\mathrm{N}$ determines the theoretical number of genetic regulators directly. The reason we call $\mathrm{N}$ theoretical genetic regulator number is that in the formula $2^{\mathrm{N}}=\mathrm{k}^{\prime}+1$, we cannot be certain about the number of $\mathrm{N}$ when several genetic regulator have same changing DNA expression level on their target giving merged step-levels. Assuming that a target $t$ have $n$

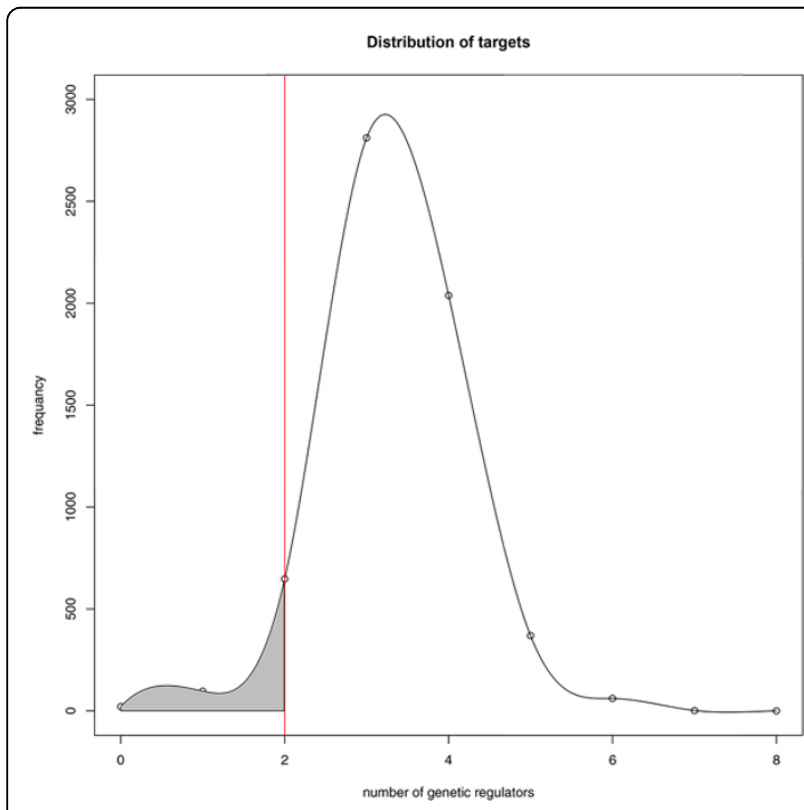

Figure $4 \mathrm{~A}$ histogram showing the distribution of regulator counts for all data in the Yeast Fit Database. The red line stands for the genetic regulators equal 2 within step levels $k^{\prime}$ from 2 to 3 . The shade area presents the genetic regulators equal or below 2 step levels excluding 0 within $k^{\prime}$ from 1 to 3 . genetic regulators $\mathrm{x}_{1}, \mathrm{x}_{2} \ldots \mathrm{x}_{\mathrm{n}}$. And $\mathrm{f}\left(\mathrm{x}_{1}, \mathrm{x}_{2} \ldots \mathrm{x}_{\mathrm{n}}\right)$ stands for step levels due to the combined genetic regulatory effect on $t$. The states of $\times$ can be either 1 or 0 to indicate whether $x$ is activated or not. The number of target's step-levels $f_{t}\left(x_{1}, x_{2} \ldots x_{n}\right)$ is greater than $n: f_{t}\left(x_{1}, x_{2} \ldots x_{n}\right) \geq n+1$. For instance, if $x_{1}, x_{2}, x_{3}$ have similar regulation ability on $t$, the $f(1,0,0), f(0,1,0)$, $f(0,0,1)$ will give a merged level. Similarly the $f(0,1,1)$, $f(1,0,1), f(1,1,0)$ will give another merged level. In this case, the step levels of $\mathrm{t}$ is $4=\mathrm{n}+1$ that stands for four levels of $f(0,0,0), f(1,0,0), f(1,1,0)$ and $f(1,1,1)$. Hence, the $\mathrm{N}$ will be 2 meaning that our predicted targets of 2 genetic regulators will contain some targets of 3 genetic regulators in extreme case. And the mis-clustering will be more severe when applied for prediction of targets with more genetic regulators.

\section{Identification of activation/inhibition genetic regulatory relationship details}

According to the Pearson Correlation model of Activation/Inhibition, 89 targets were selected with 3473 activation pairs in initial 453 de-centric targets generated from SLDR and 94 targets were selected with 2271 inhibition pairs in initial 453 de-centric targets generated from SLDR. After applying the criteria of rejection, we found 83 targets with 3190 activation pairs and 93 targets with 2128 inhibition pairs. After ordering all 5318 regulation pairs in the genome for each target by finding $\max f(t, x):\left|\mathrm{R}_{\mathrm{o}}-\mathrm{R}_{\mathrm{h}}\right|$ and choosing top 10 high pairs, 610 activation pairs (Additional file 1) and 494 inhibition pairs (Additional file 2) were selected. 176 targets candidates were queried in Yeast Fitness Database, 64 targets were identified afterwards. Among them, 33 targets with 115 activation pairs and 31 targets with 97 inhibition pairs were validated. (Table 3).

The contingency table of genetic regulatory relationship network is shown in (Table 4). The top 10 high $\left|R_{o}-R_{h}\right|$ genetic regulatory pairs is 1104 within 212 pairs been

Table 3 Summary Statistics for validated de-centric targets and genetic regulatory relationship identified

\begin{tabular}{ccccccc}
\hline \multicolumn{3}{c}{ Activation } & \multicolumn{3}{c}{ inhibition } \\
\hline & Validated & total & rate & validated & total & rate \\
\hline pairs & $115(33)$ & $610(83)$ & $19 \%(40 \%)$ & $97(31)$ & $494(93)$ & $20 \%(33 \%)$ \\
\hline
\end{tabular}

Table 4 A distribution of significant genetic regulatory relationship according to rank groups

\begin{tabular}{rcc}
\hline & top 10 & Random \\
\hline validated & 212 & 433 \\
un-validated & 892 & 6956 \\
total & 1104 & 7389 \\
\hline
\end{tabular}




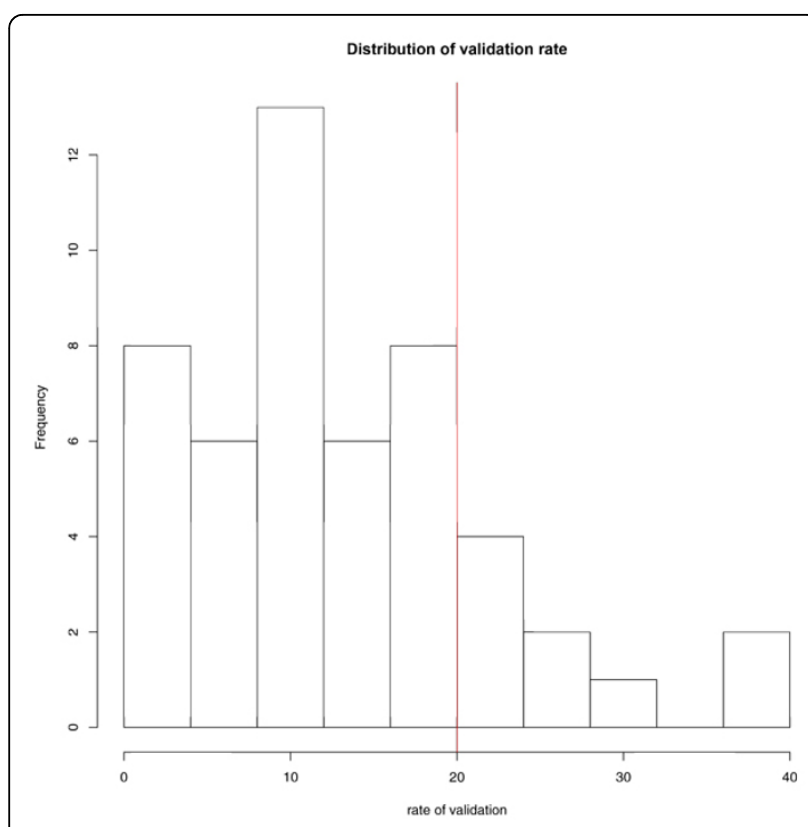

Figure 5 The distribution of validation rate of randomly chosen pairs. The red line drawn at rate of validation $=20$ refers to a $20 \%$ validation ratio for top $10\left|R_{o}-R_{h}\right|$ pairs. 9 out of 50 is above to $18 \%, 41$ out of 50 is below $82 \%$.

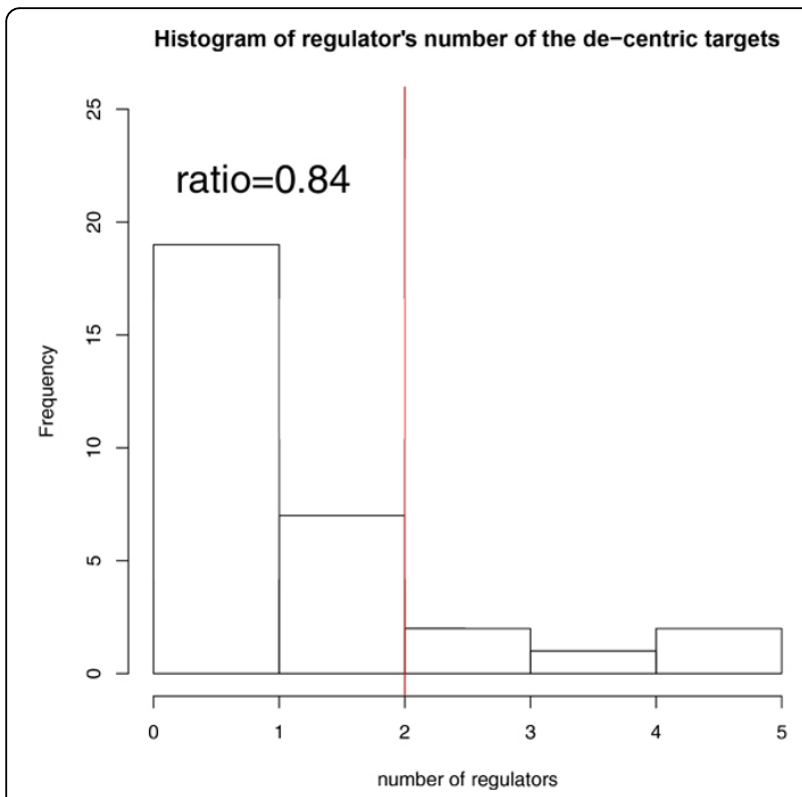

Figure 6 A histogram showing the distribution of regulator counts among all the de-centric gene targets. A red line shows the ratio of the target's number which regulators' number equal or below 2 to the total target's number is 0.84 .

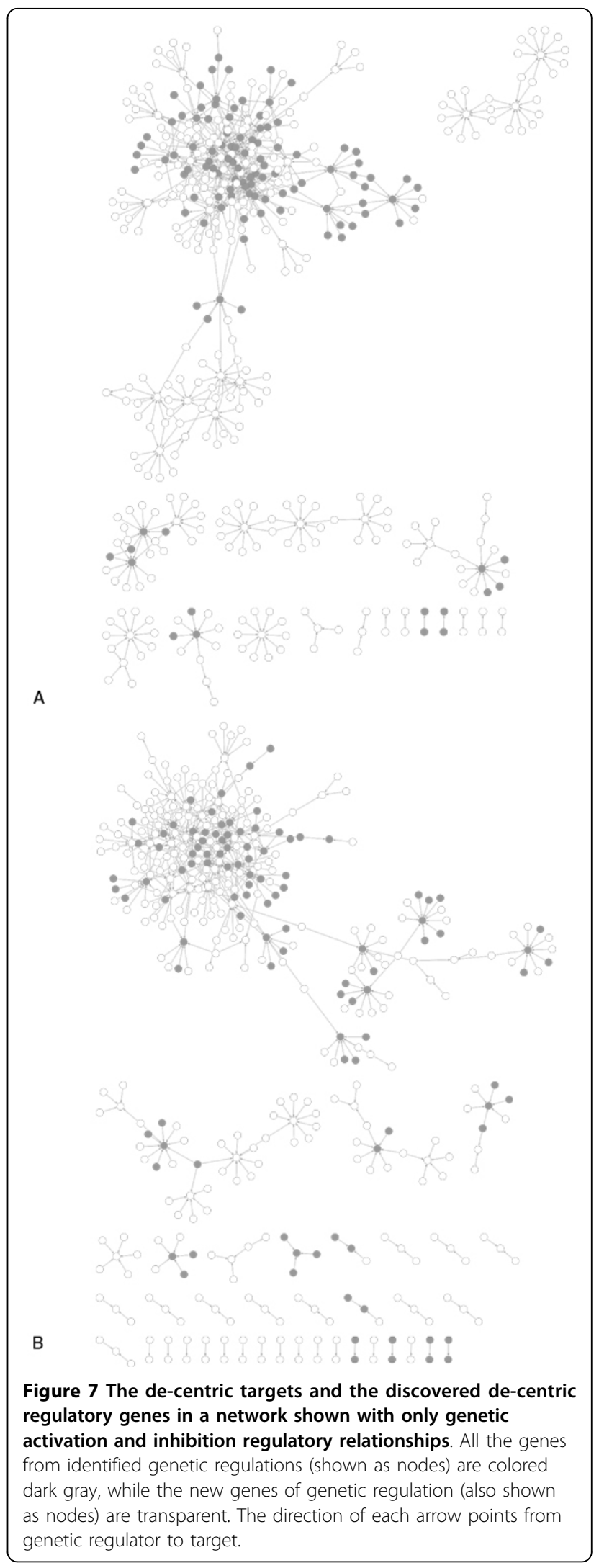




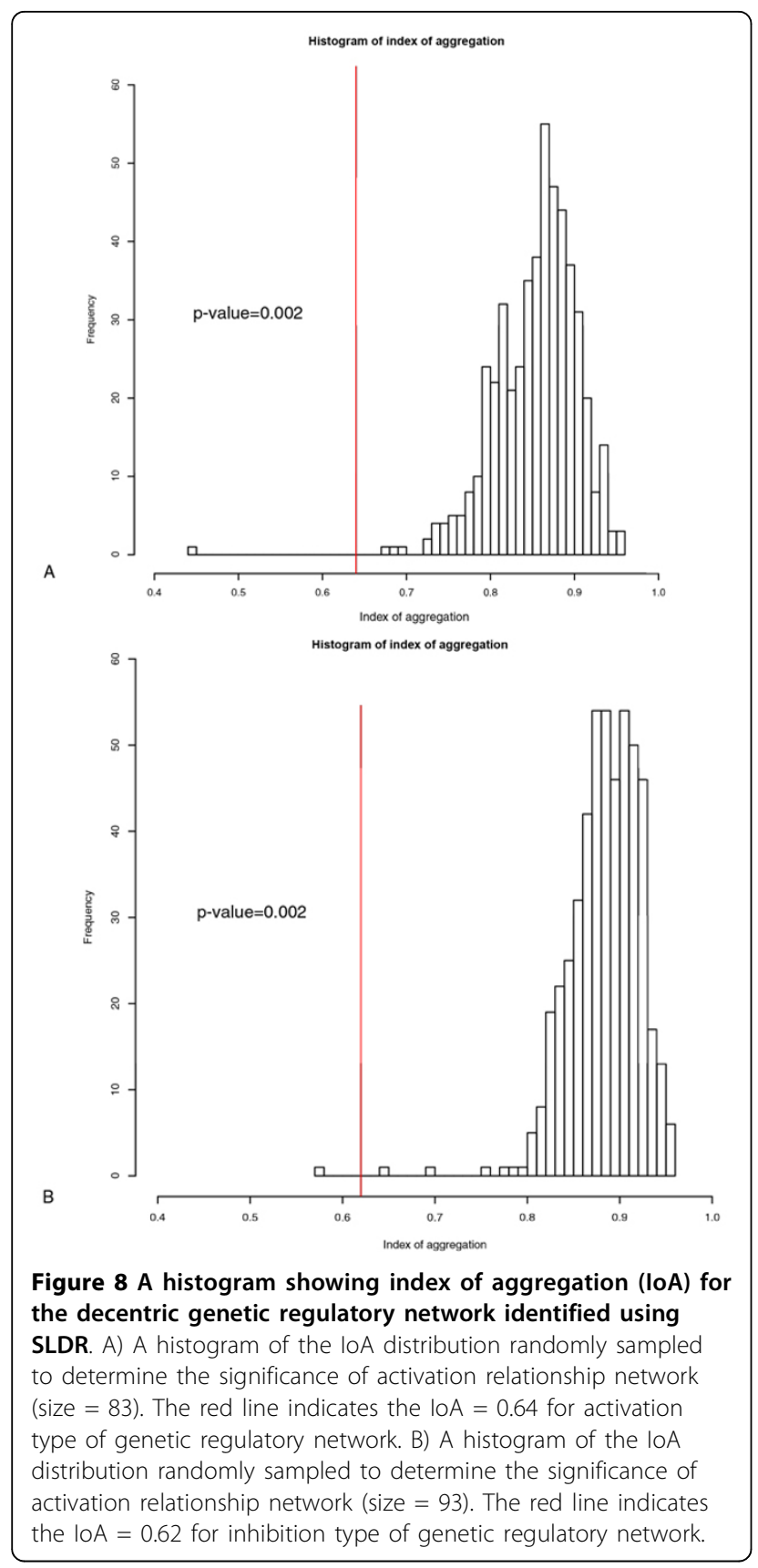

validated. The randomly chosen pairs is 7389 within 433 pairs been validated. The $\mathrm{p}$-value of $7.44 \mathrm{e}^{-43}$ showed the significant difference between top $10\left|R_{\mathrm{o}}-\mathrm{R}_{\mathrm{h}}\right|$ pairs and randomly chosen pairs. We also preformed resample of randomly chosen pairs (the same size to $10\left|R_{\mathrm{o}}-\mathrm{R}_{\mathrm{h}}\right|$ pairs: 1104) with Pearson Correlation cut off 0.2 and 0.8 for 50 times and generated the distribution of validation rate (Figure 5). $18 \%$ of pairs above $20 \%$ validation rate indicated that the top $10\left|R_{\mathrm{o}}-\mathrm{R}_{\mathrm{h}}\right|$ pairs is more significant than randomly one.

The 174 de-centric genetic regulatory targets ( 81 active targets, 91 inhibited targets, 2 in both active targets set and inhibited targets set) predicted by SLDR were queried in the Yeastract Database (http://www.yeastract.com/ index.php). The evidence of DNA binding and expression in database's documents covers 31 of 173 de-centric genetic regulatory targets. The 142 residue de-centric genetic regulatory targets haven't been discovered probably due to the limited experimental techniques. The number of regulators of 31 de-centric genetic regulatory targets is small and not above 5 . The ratio of the target's number with equal or below 2 regulators to the total target's number is 0.84 (Figure 6). Hence, SLDR is able to detect the de-centric genetic regulatory targets confirmed by the Yeastract Database.

\section{Construction of de-centric target-regulator network}

We constructed the network of the 610 activation pairs and 494 inhibition pairs (Figure 7). 112 targets were found to be new candidates with its genetic regulator. The hub nodes with high-connectivity linked to the majority of nodes to form a main structure of network. The peripheral nodes with low-connectivity formed relativity small sub-networks or even one-to-one model. The ratio of the size of the largest sub-network that exists in this network to the size of this network, we defined as index of aggregation, reflect hub-nodes weight. The index of aggregation in the activation genetic regulatory relationship is $64 \%$. The index of aggregation in the inhibition genetic regulatory relationship is $62 \%$.

Table 5 Effect of introducing noise in the results on the network index of aggregation (showing p-value changes)

\begin{tabular}{|c|c|c|c|c|c|c|c|c|c|}
\hline \multicolumn{2}{|c|}{ Noise } & \multirow{2}{*}{$\begin{array}{c}\mathbf{0} \\
59\end{array}$} & \multirow{2}{*}{$\begin{array}{c}5 \\
36\end{array}$} & \multirow{2}{*}{$\begin{array}{l}10 \\
20\end{array}$} & \multirow{2}{*}{$\begin{array}{l}15 \\
22\end{array}$} & \multirow{2}{*}{$\begin{array}{c}20 \\
9\end{array}$} & \multirow{2}{*}{$\begin{array}{c}30 \\
7\end{array}$} & \multirow{2}{*}{$\begin{array}{c}50 \\
6\end{array}$} & \multirow{2}{*}{$\begin{array}{c}70 \\
3\end{array}$} \\
\hline positive & regulator & & & & & & & & \\
\hline & $p$-value & $0.002^{* *}$ & $0.042^{* *}$ & 0.216 & 0.61 & 0.294 & 0.14 & 0.242 & 0.292 \\
\hline & Variance & 0.0025 & 0.0166 & 0.0313 & 0.0174 & 0.0216 & 0.0201 & 0.0188 & 0.019913 \\
\hline \multirow[t]{3}{*}{ negtive } & regulator & 48 & 36 & 19 & 17 & 9 & 4 & 4 & 4 \\
\hline & p-value & $0.002^{* *}$ & $0.014^{* *}$ & $0.078^{*}$ & 0.124 & $0.088^{*}$ & 0.496 & 0.504 & 0.404 \\
\hline & Variance & 0.0016 & 0.0091 & 0.0254 & 0.0196 & 0.0277 & 0.0188 & 0.0145 & 0.014751 \\
\hline
\end{tabular}




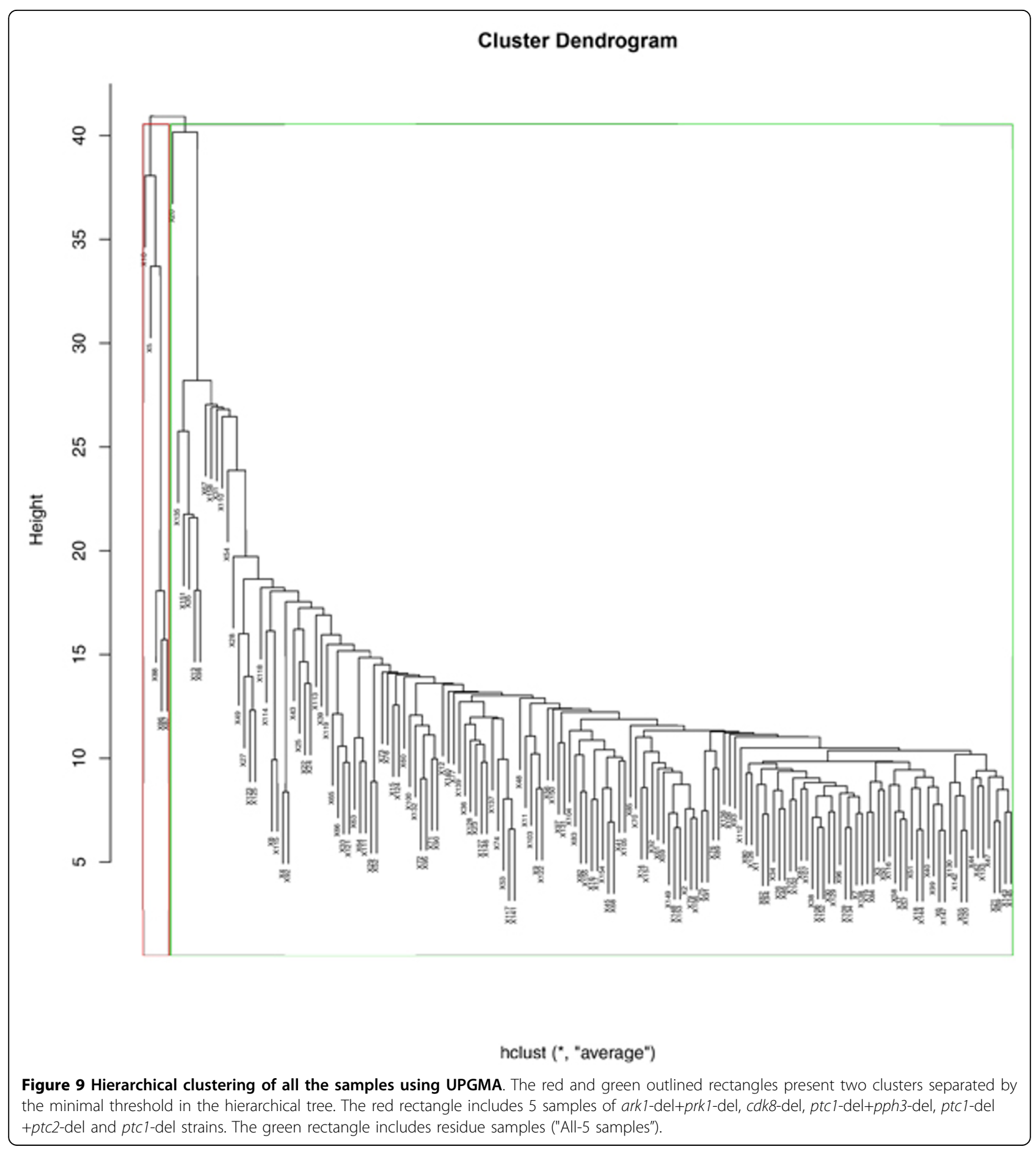

Table 6 Validated de-centric targets and genetic regulatory relationship

\begin{tabular}{lrrrrrrr}
\hline & \multicolumn{3}{c}{ activation } & \multicolumn{3}{c}{ inhibition } \\
\hline & validated & total & rate & validated & Total & rate \\
\hline Pairs & 115 & 610 & $19 \%$ & 97 & 494 & $20 \%$ \\
\hline pairs(after cluster) & 244 & 999 & $24 \%$ & 110 & 805 & $14 \%$ \\
\hline Change & $(+) 129$ & $(+) 389$ & $(+) 5 \%$ & $(-) 13$ & & $(-) 311$ & $(-) 6 \%$ \\
\hline
\end{tabular}

\section{Significance evaluation of de-centric genetic regulatory} relationship identified

The empirical distribution of the index of aggregation was obtained after 500 random re-samplings (Figure 8). Only 1 run out of 500 resulted in an index of aggregation value greater than $99.8 \%$ in both de-centric genetic activation regulatory network and de-centric genetic 
Table 7 A Comparison between all the samples and the All-5 Samples (5 deleted after hierarchical clustering) to show changes of $p$-values for the network's index of aggregation

\begin{tabular}{llcr}
\hline & noise & total samples & 5 samples deletion \\
\hline \multirow{2}{*}{ positive } & regulator & 59 & 132 \\
\cline { 2 - 4 } & p-value & $\mathbf{0 . 0 0 2 ^ { * * }}$ & 0.186 \\
\cline { 2 - 4 } & variance & 0.002532727 & 0.002405613 \\
\hline \multirow{2}{*}{ negative } & regulator & 48 & 135 \\
\cline { 2 - 4 } & p-value & $\mathbf{0 . 0 0 2}$ & 0.116 \\
\cline { 2 - 4 } & variance & 0.001573338 & 0.006110383
\end{tabular}

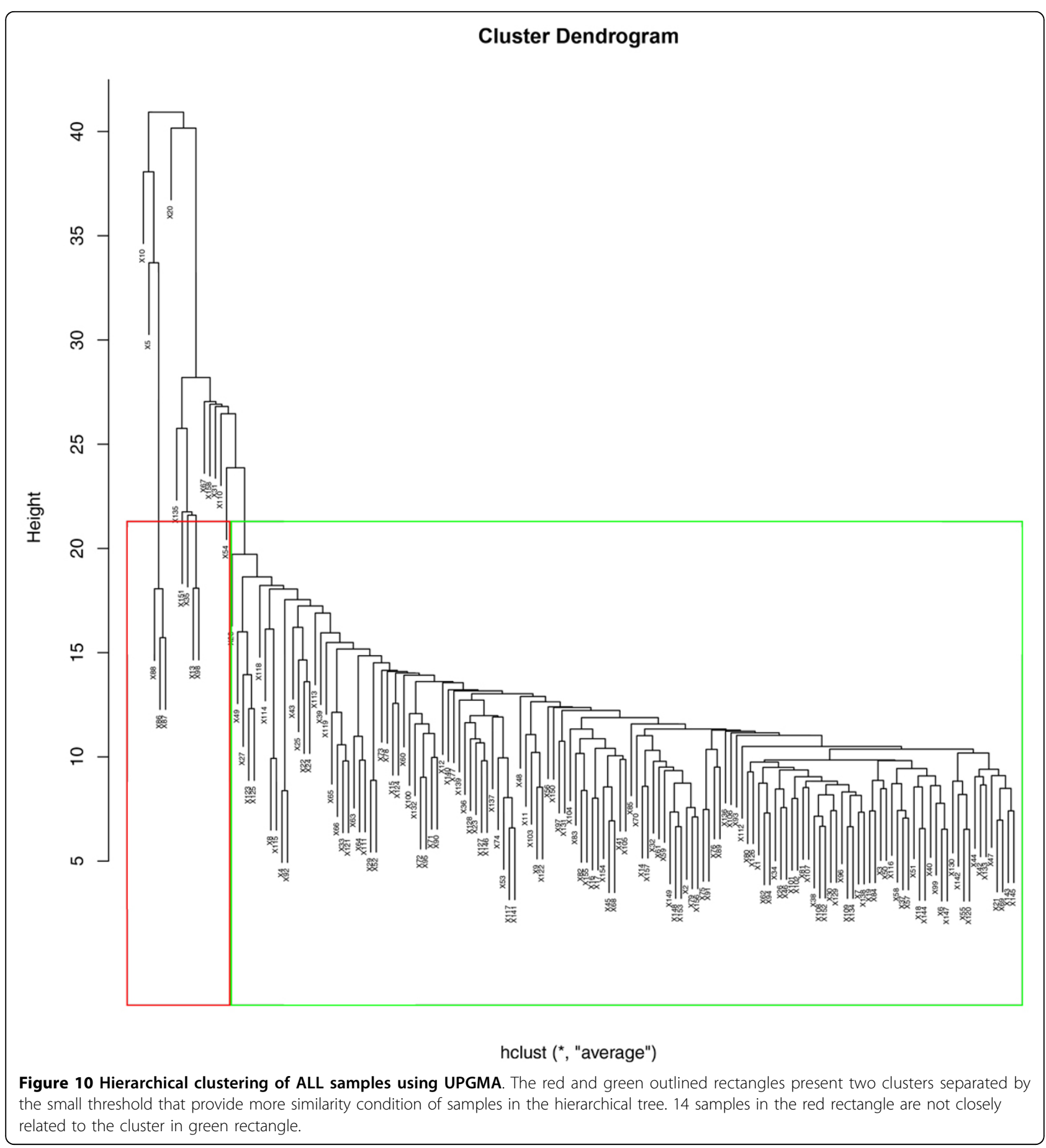


inhibition regulatory network. Therefore, the p-value of the index of aggregation is 0.002 . It is not surprising to observe such a significant result since the results are selected in a way that the theoretical genetic regulator is equal or below 2 . Hence, the aggregation test confirmed result of clustering of gene expression profiles and transcription factor binding site pattern matching is significant in discovering de-centric genetic regulatory relationship.

\section{Robustness of the genetic regulatory network}

After we introduced the noise by randomly changing $5 \%, 10 \%, 15 \%, 20 \%, 30 \%, 50 \%, 70 \%$ of total samples, the p-value of network aggregation increase significantly (Table 5). The aggregation p-value below 0.05 is high significant with bold type. The aggregation p-value below 0.1 and above 0.05 is significant with bold type. The aggregation p-value above 0.1 is weak significant or no significant.

The result shows that the network has resistance of 5\% noise. However, along the noise increase, a significant weakening appears on detecting the de-centric regulators and finding potential targets using SLDR. In the randomly generated networks by small number of targets, the network aggregation test tends to be bias reflected on the increased variance.

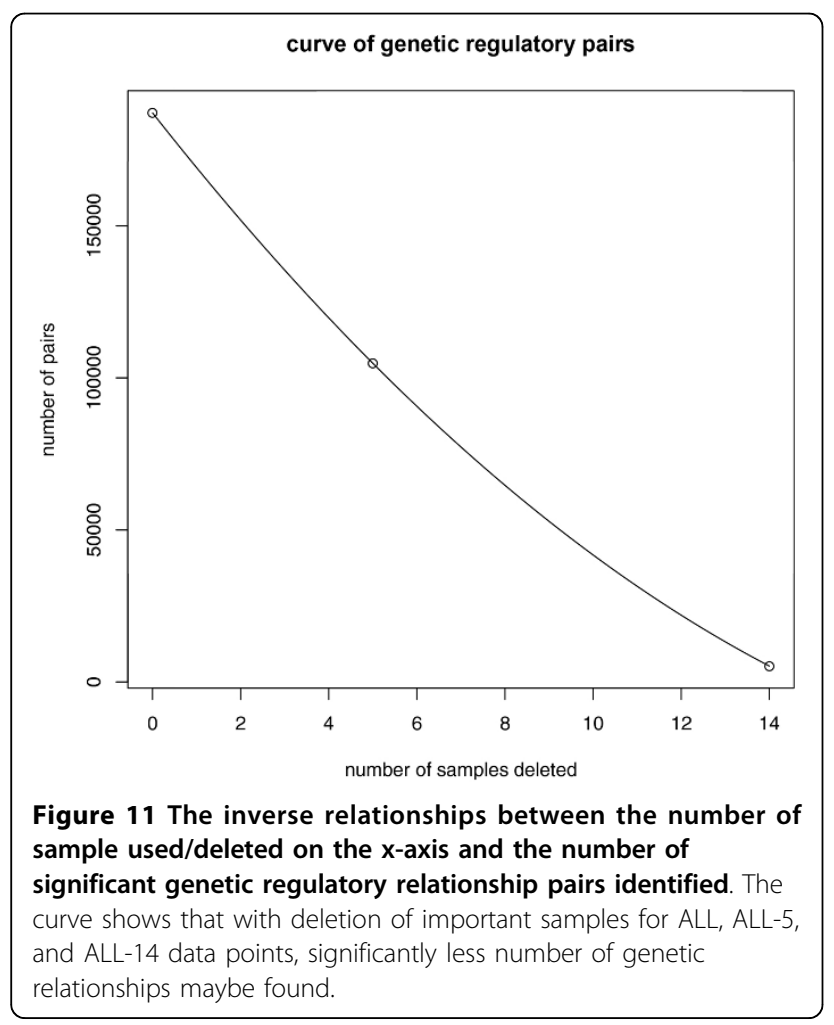

\section{Decisive cluster of samples}

Applying UPGMA clustering, the samples were divided into two groups using a minimal threshold in the hierarchical tree. We chose the majority of samples which has similarity conditions clustered in green rectangle (Figure 9) and generated the de-centric genetic regulatory targets and pairs applying SLDR method. After we constructed the network of the 999 activation pairs to targets and 805 inhibition pairs,163 targets were found to be new candidates with its genetic regulator. The index of aggregation in the activation genetic regulatory

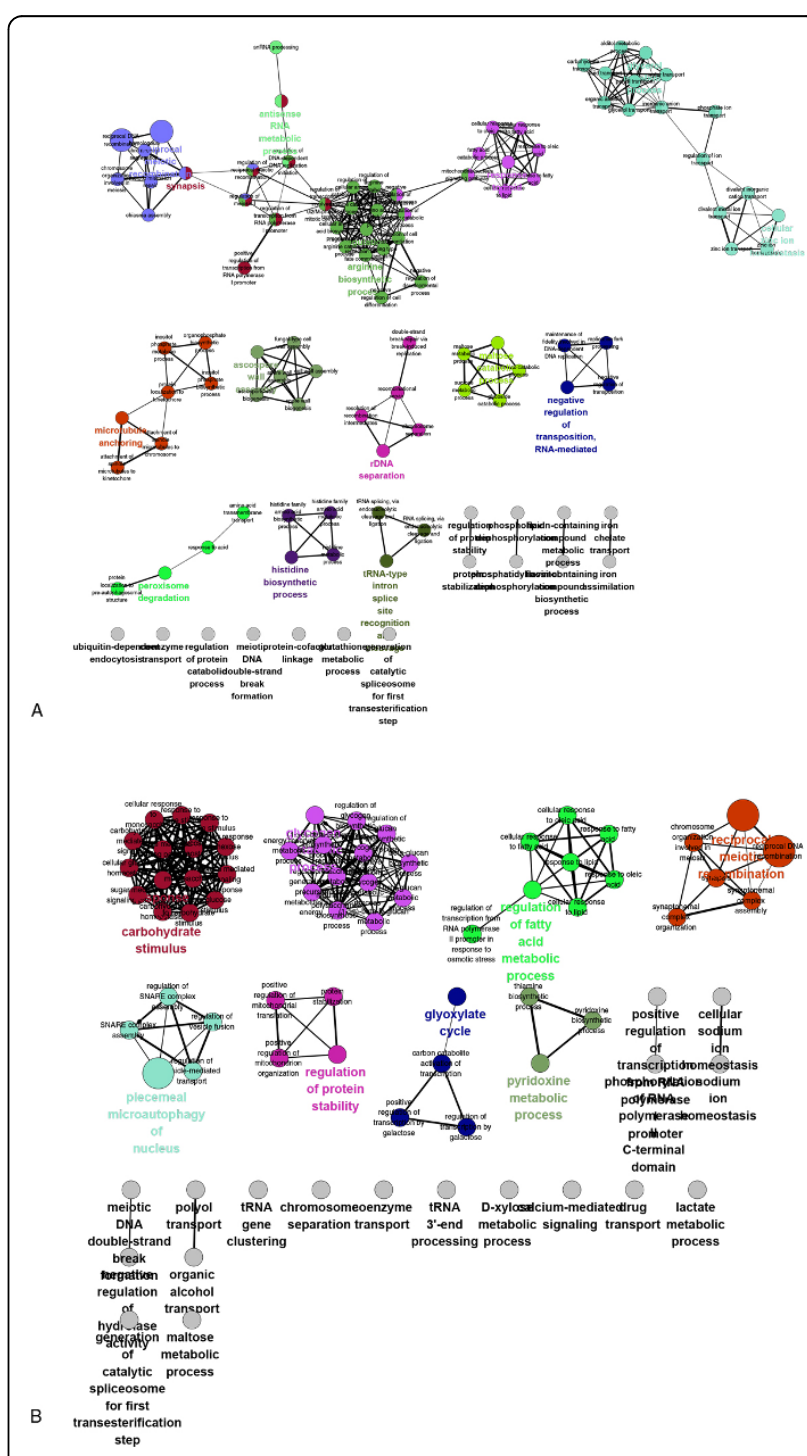

Figure 12 The biology process groups of de-centric genetic regulatory networks. A) The biology process groups of genetic activation regulatory networks. B) The biology process groups of genetic activation regulatory networks. The colorful nodes stand for biology processes. The biology processes grouped together with same color by measuring the similarity using Kappa scores. The groups consist of grey nodes were not found a certain and consensus function. 
relationship is $78 \%$. The index of aggregation in the inhibition genetic regulatory relationship is $81 \%$.

According to the Pearson Correlation model of Activation/Inhibition, 132 targets with 8558 activation pairs were selected in initial 744 de-centric regulatory targets generated from SLDR and 135 targets with 4486 inhibition pairs were selected in initial 744 de-centric targets generated from SLDR. After ordering all regulation pairs in the genome for each target by finding $\max f(t, x):\left|\mathrm{R}_{\mathrm{o}}-\mathrm{R}_{\mathrm{h}}\right|$ and choosing top 10 high pairs, 545 activation pairs and 482 inhibition pairs were selected. (Index 2) 268 targets candidates were queried in Yeast Fitness Database, 105 targets were identified afterwards. Among them, 70 targets with 244 activation pairs and 35 targets with 110 inhibition pairs were certificated. Comparing to the previous genetic regulatory de-centric pairs generated from un-delete samples, the validated activation pairs increase 129( i.e. 5\%) while validated inhibition pairs decrease 13 (i.e. 6\%). (Table 6).

The empirical distribution of the index of aggregation was obtained after 500 random re-samplings. 93 runs out of 500 resulted in an index of aggregation value greater than $81.4 \%$ in de-centric genetic activation regulatory network and de-centric genetic inhibition regulatory network and 58 runs out of 500 resulted in an index of aggregation value greater than $88.4 \%$ in de-centric genetic activation regulatory network and de-centric genetic inhibition regulatory network. Therefore, the $\mathrm{p}$-value of the index of aggregation in de-centric genetic activation regulatory network is 0.186 and the p-value of the index of aggregation in de-centric genetic inhibition regulatory network is 0.116 .

The result (Table 7) shows that the de-centric regulatory network generated without 5 samples of ark1-del+prk1del, $c d k 8$-del, $p t c 1$-del+pph3-del, $p t c 1-\mathrm{del}+p t c 2$-del and $p t c 1$-del strains would entirely lose ability to detect the decentric targets. In complete samples SLDR method, the network has $5 \%$ resistance of noise. Here we selected 5 samples that is $3 \%$ of entire samples deletion. Then the aggregation cannot detect de-centric targets. It reveals that the cluster of 5 samples of ark1-del+prk1-del, $c d k 8$-del, $p t c 1$-del $+p p h 3$-del, $p t c 1$-del $+p t c 2$-del and $p t c 1$-del strains is a decisive cluster.

In order to see the decisive samples effect on regulatory relationship, we lifted the threshold in the UPGMA hierarchical tree. We chose the more strict similarity of samples which were clustered in green rectangle and deleted the decisive cluster of 16 samples in red rectangle (Figure 10). However, we generated the de-centric genetic regulatory targets and pairs applying SLDR method in the result of only 4 activate genetic regulatory targets with 6 pairs and 3 inhibit genetic regulatory targets with 9 pairs. Hence, the decisive cluster also has effect on finding de-centric pairs, which confirmed by a significant sharp drop in finding the genetic regulatory pairs of regulators to targets by deletion of 16 samples (Figure 11).

\section{The biology process groups of de-centric genetic regulatory networks}

The biology process of de-centric genetic regulatory networks were explored and grouped by ClueGo (Figure 12). In the de-centric activation genetic regulatory network, 14 functional groups were found (Table 8A). 4 significant function with above 10 gene numbers in the groups: synapsis, reciprocal meiotic recombination, glycerol metabolic process, and regulation of arginine biosynthetic process. In the de-centric inhibition regulatory network, 7 functional groups were found (Table 8B). 2 significant function with above 10 gene numbers in the groups: piecemeal microautophagy of nucleus, and reciprocal meiotic recombination. These 5 (3 in activation, 1 in inhibition, 1 in common) significant function indicate that the de-centric genetic regulatory networks were

Table 8 The biology process group lists of de-centric genetic regulatory networks.

\begin{tabular}{lll}
\hline A. & & \\
\hline Function & Groups & Gene \\
& numbers \\
\hline ascospore wall assembly & Group0 & 6 \\
peroxisome degradation & Group1 & 8 \\
synapsis & Group2 & $\mathbf{1 1}$ \\
tRNA-type intron splice site recognition and & Group3 & 2 \\
cleavage & & \\
reciprocal meiotic recombination & Group4 & $\mathbf{2 0}$ \\
histidine biosynthetic process & Group5 & 2 \\
maltose catabolic process & Group6 & 3 \\
glycerol metabolic process & Group7 & $\mathbf{1 5}$ \\
regulation of arginine biosynthetic process & Group8 & $\mathbf{1 6}$ \\
antisense RNA metabolic process & Group9 & 6 \\
cellular zinc ion homeostasis & Group10 & 9 \\
negative regulation of transposition, RNA- & Group11 & 3 \\
mediated & & \\
rDNA separation & Group12 & 8 \\
microtubule anchoring & Group13 & 5 \\
response to lipid & Group14 & 7 \\
\hline B. & & \\
\hline Function & Groups & Gene \\
\hline pyridoxine metabolic process & numbers \\
regulation of fatty acid metabolic process & Group1 & 3 \\
piecemeal microautophagy of nucleus & Group2 & $\mathbf{1 0}$ \\
glyoxylate cycle & Group3 & 5 \\
regulation of protein stability & Group4 & 3 \\
breciprocal meiotic recombination & Group5 & $\mathbf{1 2}$ \\
glycogen metabolic process & Group6 & 8 \\
response to carbohydrate stimulus & Group7 & 2 \\
\hline & & \\
\hline
\end{tabular}




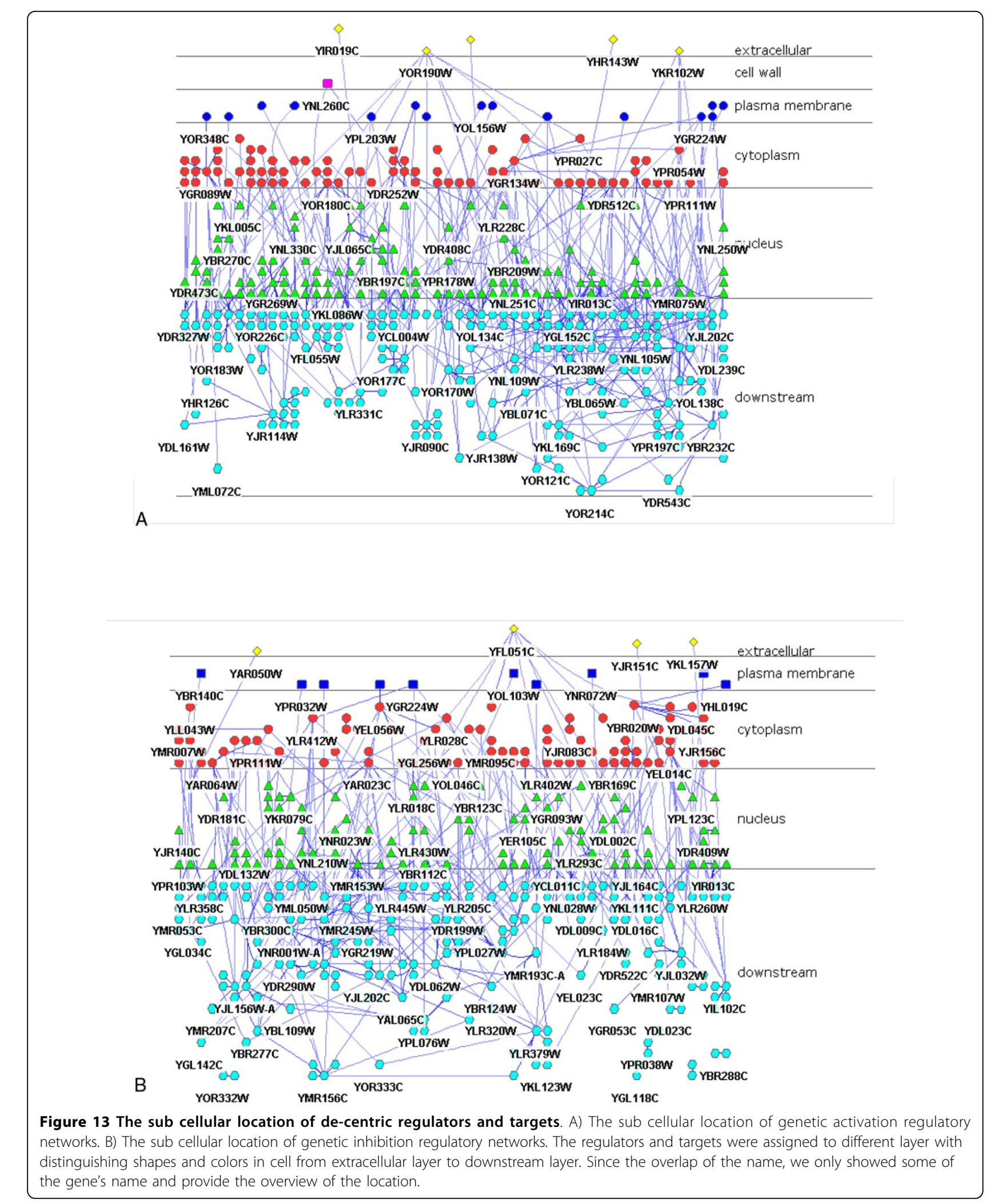


functional and involved in basic and fundamental biology process in cells, especially like reciprocal meiotic recombination, which found in both de-centric activation and inhibition networks.

\section{The sub cellular location of de-centric regulators and targets in cell}

The sub cellular location of de-centric regulators and targets showed a complex regulatory networks through the cells (Figure 13). In both de-centric activation regulators and targets networks, and de-centric activation regulators and targets networks, the regulatory pathways signals were cascade in cell - extracellular proteins and membrane receptors at the top, adapter proteins in the cytoplasm, and nuclear proteins and pathway-regulated genes at the bottom.

\section{Discussion}

In this study, we discovered 112 significant new genetic regulatory targets with functional genomics data set derived from 158 viable protein kinase/phosphatase deletion experiments. For each new target, top 10 highranking suspected genetic regulatory pair candidates are also given. This genetic regulatory relationship of regulators to targets can include much more than the direct regulation and control, because genetic regulators could sit in the far upstream of its targets.

We developed SLDR to identify candidate genetic regulators particularly of none-hub gene targets. Unlike a wholetranscriptome profiling based correlation techniques, SLDR can efficiently detect signal levels as "clustered conditions", each of which correspond to a "step-level" of target gene perturbed/regulated by a set of combinatorial states of regulator genes. Because the correlation is performed at the "step levels", SLDR can therefore screen, identify, and rank potential regulation relationship of genetic regulators to targets. The prediction pairs can be validated reasonably well by data from the Yeast Fitness Database, although the overlap is not high due to incomplete coverage of the database. Note that our method of finding genetic regulatory relationship of regulators to targets relies on Pearson Correlation at the moment. Future extension of this work could explore additional correlation techniques such as using Goodman and Kruskal's gamma correlation coefficient or Spearman's rank correlation coefficient to compare their impact on prediction performance.

In summary, our study demonstrated a new direction to identify genetic regulatory relationships. If applied broadly, the technique could yield many new data worth biological investigations. Such relationships, although indirect, may help construct biological signaling network to overcome coverage issues facing network biology models today. Future directions to make SLDR an easyto-use software package and develop databases to capture the results are under development. By applying SLDR to human gene expression perturbation data, we believe our framework may also be extended to provide complementary insights on human complex biological systems and disease network biology.

\section{Additional material}

\section{Additional file 1:}

Additional file 2:

\section{Competing interests}

Jake Y. Chen is the founder and chairman of Medeolinx, LLC of Indianapolis, IN. All authors declare no competing interests in connection with this work.

\section{Acknowledgements}

This work and publication was supported in part by research funding obtained by Jake Chen at Wenzhou Medical University from China and at Indiana University School of Informatics and Computing. We also thank the generous support of Capital Normal University, China to provide the initial resources that led to the conception of this work.

This article has been published as part of BMC Bioinformatics Volume 15 Supplement 11, 2014: Proceedings of the 11th Annual MCBIOS Conference. The full contents of the supplement are available online at http://www. biomedcentral.com/bmcbioinformatics/supplements/15/S11.

\section{Authors' details}

${ }^{1}$ Institute of Biopharmaceutical Informatics and Technology, Wenzhou Medical University, Wenzhou, Zhejiang Province, China. ${ }^{2}$ School of Informatics, Indiana University, Indianapolis, IN 46202, USA. ${ }^{3}$ Indiana Center for Systems Biology and Personalized Medicine, Indiana University, Indianapolis, IN 46202, USA. ${ }^{4}$ Bioinformatics lab, Capital Normal University, Beijing, China.

Published: 21 October 2014

\section{References}

1. James CG, Appleton CT, Ulici V, Underhill TM, Beier F: Microarray analyses of gene expression during chondrocyte differentiation identifies novel regulators of hypertrophy. Molecular biology of the cell 2005, 16(11):5316-5333.

2. Lorenz WW, Alba R, Yu YS, Bordeaux JM, Simoes M, Dean JF: Microarray analysis and scale-free gene networks identify candidate regulators in drought-stressed roots of loblolly pine (P. taeda L.). BMC genomics 2011, $12: 264$.

3. Wang W, Cherry JM, Botstein D, Li H: A systematic approach to reconstructing transcription networks in Saccharomycescerevisiae. Proceedings of the National Academy of Sciences of the United States of America 2002, 99(26):16893-16898.

4. Barabasi AL, Gulbahce N, Loscalzo J: Network medicine: a network-based approach to human disease. Nature reviews Genetics 2011, 12(1):56-68.

5. Snyder M, Gallagher JE: Systems biology from a yeast omics perspective. FEBS letters 2009, 583(24):3895-3899.

6. Goodson HV, Anderson BL: Synthetic Lethality Screen Identifies a Novel Yeast. The Journal of Cell Biology 1996, 133(6):1277-1291.

7. Landt Stephen G, Marinov Georgi K, Anshul Kundaje, Pouya Kheradpour: ChIP-seq guidelines and practices of the ENCODE and modENCODE consortia. Cold Spring Harbor Laboratory Press 2012, , 22: 1813-1831.

8. Chen J, Shen C: MINING ALZHEIMER DISEASE RELEVANT PROTEINS FROM INTEGRATED PROTEIN INTERACTOME DATA. Pacific Symposium on Biocomputing 2006, 11:367-378.

9. Yeh RF, Lim LP, Burge CB: Computational inference of homologous gene structures in the human genome. Genome research 2001, 11(5):803-816.

10. Audic S, Claverie J-M: The Significance of Digital Gene Expression Profiles. Genome research 1997, 7:986-995. 
11. Gerhard MGM: A system for pattern matching applications on biosequences. Comput Appl Biosci 1993, 9(3):299-314.

12. Huang N, Jacobson MP: Physics-based methods for studying proteinligand interactions. Current Opinion in Drug Discovery \& Development 2007, 10(3):325-331.

13. Wan P, Yue Z, Xie Z, Gao Q, Yu M, Yang Z, Huang J: Mechanisms of Radiation Resistance in Deinococcus radiodurans R1 Revealed by the Reconstruction of Gene Regulatory Network Using Bayesian Network Approach. J Proteomics Bioinform 2013, 6(7)

14. van Wageningen $S$, Kemmeren $P$, Lijnzaad $P$, Margaritis T, Benschop JJ, de Castro IJ, van Leenen D, Groot Koerkamp MJ, Ko CW, Miles AJ, et al: Functional overlap and regulatory links shape genetic interactions between signaling pathways. Cell 2010, 143(6):991-1004.

15. Hillenmeyer ME, Fung E, Wildenhain J, Pierce SE, Hoon S, Lee W, Proctor M, St Onge RP, Tyers M, Koller D, et al: The chemical genomic portrait of yeast: uncovering a phenotype for all genes. Science 2008, 320(5874):362-365

16. Bindea G, Mlecnik B, Hackl H, Charoentong P, Tosolini M, Kirilovsky A, Fridman W-H, Pagès F, Trajanoski Z, Galon J: ClueGO: a Cytoscape plug-in to decipher functionally grouped gene ontology and pathway annotation networks. Bioinformatics 2009, 25(8):1091-1093.

17. Idener UG, tter MMn, ller GK, Strack N, Helden JV, Lemer C, Richelles J, Wodak SJ, García-Martínez J, Peérez-Ortín3 JE, et al: CYGD: the Comprehensive Yeast Genome Database. Nucleic acids research 2005, 33(Database):10-1093.

18. Barsky A, Gardy JL, Hancock REW, Munzner T: Cerebral: a Cytoscape plugin for layout of and interaction with biological networks using subcellular localization annotation. Bioinformatics 2007, 23(8):1040-1042.

doi:10.1186/1471-2105-15-S11-S1

Cite this article as: Yue et al: SLDR: a computational technique to identify novel genetic regulatory relationships. BMC Bioinformatics 2014 15(Suppl 11):S1.

\section{Submit your next manuscript to BioMed Central and take full advantage of:}

- Convenient online submission

- Thorough peer review

- No space constraints or color figure charges

- Immediate publication on acceptance

- Inclusion in PubMed, CAS, Scopus and Google Scholar

- Research which is freely available for redistribution

Submit your manuscript at www.biomedcentral.com/submit
Biomed Central 